\title{
Messenger RNA expression profiling using DNA microarray technology: Diagnostic tool, scientific analysis or un-interpretable data? (Review)
}

\author{
MOIRA S. WALKER and THOMAS A. HUGHES \\ YCR and Liz Dawn Pathology and Translational Sciences Centre, Leeds Institute of Molecular Medicine, \\ Wellcome Trust Brenner Building, St James's University Hospital, University of Leeds, Leeds LS9 7TF, UK
}

Received May 21, 2007; Accepted July 2, 2007

\begin{abstract}
Analysis of the transcriptome using DNA microarrays has become a standard approach for investigation of the molecular basis of human disease in both clinical and experimental settings. However, drawing conclusions from the wealth of data obtained has remained problematic. There have been difficulties with accurate reporting of results, with experimental reproducibility and with identifying and interpreting the biologically relevant information. In this review we discuss the successful use of DNA microarray technology in molecular medical research, and we highlight methods of addressing the issues of both reproducibility and biological interpretation.
\end{abstract}

\section{Contents}

1. Introduction

2. Applications of DNA microarrays

3. Variability in results

4. Additional regulatory mechanisms in gene expression

5. Conclusions

\section{Introduction}

The sequencing of the human genome inspired the scientific community to investigate the functions of the newly sequenced, and in many cases newly discovered, genes. A number of methods were employed, including DNA microarrays for analysis of messenger RNA (mRNA) expression. This

Correspondence to: Dr Thomas A. Hughes, YCR and Liz Dawn Pathology and Translational Sciences Centre, Leeds Institute of Molecular Medicine, Wellcome Trust Brenner Building, St James's University Hospital, University of Leeds, Leeds LS9 7TF, UK

E-mail: t.hughes@leeds.ac.uk

Key words: microarray technology, transcriptome technique, while stopping short of allowing functions to be assigned to individual genes, promised to provide a powerful method of simultaneously assessing gene activity for huge sets of genes in multiple samples, thereby allowing conclusions about the functions and importance of particular genes and pathways in various cellular and physiological situations. The purpose of this review is to assess to what extent this promise has been met and in which scientific fields.

$m R N A$ expression profiling. While the genome is broadly common to all the cells in an organism and is generally static over its lifetime, the transcriptome is highly dynamic and changes in response to external stimuli and in disease. Analysis of the transcriptome has been seen as critical in understanding cell behaviour. Messenger RNA expression profiling is the term given to analysis of the transcriptome by simultaneous quantification of the levels of mRNAs expressed from large numbers of genes. These profiles potentially contain information concerning the total transcriptional output of the cell, the principal determinant of protein levels and therefore of cellular phenotype (1). The size and complexity of this information far outstrips that which was previously achievable using traditional techniques such as Northern blot analysis or quantitative reverse transcriptase-PCR. It should be noted that mRNAs are, of course, only an intermediate in the synthesis of proteins from the information encoded in DNA, but in most cases it is reasonable to assume that mRNA levels are representative of protein levels (see section 4).

Development of array technology. The introduction of DNA microarray technology in 1995 (2) was made possible by a number of key concepts. In 1975, Edwin Southern, now Professor of Biochemistry (Oxford University, UK), proposed that hybridisation between complementary strands of nucleic acids, one labelled to allow detection, and the other immobilised on a matrix, could be used to interrogate genetic material (3). This principle allowed the development of the Southern blot, in which a mixed pool of DNA sequences is immobilised on a membrane, and target sequences within the pool are semi-quantitatively detected using radioactively labelled DNA probes. The Southern blot can now be viewed as the first DNA array (4). Similarly, a variant technique, the Northern blot, was developed in which RNA expression from 
individual genes is analysed within total cellular RNA using gene-specific radioactive probes. The essential conceptual advance allowing array-based analysis is a switch in which the nucleic acid strand is immobilised and which is labelled for detection; for DNA microarray analysis, the probes are immobilised on the matrix and total cellular RNA is labelled. In this way, multiple probes can be localised to different areas of the matrix, and expression of multiple targets within the total pool of RNA can be examined simultaneously. Technological improvements have also been essential for the production of convenient and reproducible microarrays. These include the use of a nonporous solid support, usually glass, enabling array miniaturisation, the development of fluorescence-based detection (5), and the introduction of high-speed spatial synthesis of oligonucleotides, allowing simultaneous synthesis of many thousands of probes in situ on arrays $(6,7)$. A number of types of microarrays are in common use, which differ in their methods of manufacture and/or the probes used. Probes include either single-stranded oilgonucleotides or cDNAs, and these can be spotted or even printed onto the microarray, or, in the case of oligonucleotides, can be synthesised in situ. This evolution has now presented us with DNA microarrays that purportedly allow measurement of the expression of all human genes in a single analysis (8).

\section{Applications of DNA microarrays}

Profiling of mRNA expression using DNA microarrays has had considerable impact on biological research; this is due, in part, to the diverse range of research areas to which the technique can be applied (9). Below we describe illustrative examples of their use in three key areas.

Expression profiling of disease. The potential for highthroughput mRNA profiling to provide novel insights into the pathogenesis of human disease was quickly recognised. Insights gained have allowed improvements in diagnosis, greater understanding of the fundamental biology of diseases, and may allow improved targeting of treatments. Messenger RNA expression profiling has been successfully used for identification of subsets of tumours that are otherwise indistinguishable $(10,11)$. For example, gene expression profiling has been used to devise a gene expression signature that allows accurate diagnosis of mediastinal B-cell lymphomas (PMBL) from within the general group of nonHodgkin's lymphoma diffuse large B-cell lymphomas (DLBCL) $(12,13)$. Clinical and morphological features alone are inadequate to provide precise diagnosis of PMBL. Numerous other studies have utilised DNA microarrays for molecular profiling of tumours with the aim of stratifying the severity of the diseases, or assigning individual cases into disease subtypes (14-16). In a successful example, transcript profiles of primary breast tumours were used as prognostic reporters that outperformed the standard clinical assessment of prognosis which uses pathological parameters (17). Similarly, profiles were devised that allowed prediction of the patients most likely to benefit from adjuvant therapy $(17,18)$. Collectively, these studies provide evidence that the use of DNA microarrays can lead to improvements in our understanding of disease and in patient care. However, the relatively high costs associated with these procedures and doubts about the reproducibility of the technology (see section 3), have limited uptake of these new clinical tools.

Delineating molecular pathways. Experimentation using DNA microarrays requires no hypothesis about which genes might be of interest since expression from very large sets of genes can be examined in an unbiased manner. This means that the technology can be used to identify unexpected transcriptional targets downstream of any stage in a molecular pathway, if that stage can either be specifically activated or repressed experimentally. Practical experimental strategies include overexpressing or 'knocking down' particular proteins of interest, or using inhibitors or agonists of their function. Changes in transcription profiles can then be determined. A frequent constraint on this approach is the inability to discriminate between changes that are directly downstream of that stage in the pathway, for example direct targets of a transcription factor of interest, from changes associated with a signalling cascade involving many stages. Despite this, the strategy has been used in many hundreds of studies. An example is the investigation of the downstream effects of human hormones; a large and diverse range of genes were found to be thyroid hormone responsive and unexpectedly a large proportion were negatively regulated (19). In this way, thyroid hormone was shown to have an influence on many novel cellular functions (20). A further example is the study of targets of the transcription factor p53. p53 was known to have a critical role in the DNA damage response, but identification of the genes involved has allowed increased understanding of why the gene is so frequently mutated in cancer $(21,22)$.

Toxicogenomics. The ability to measure genome-wide changes in gene expression upon exposure to various chemicals has led to the development of a new field toxicogenomics. DNA microarrays can provide insights into the mechanisms of action of toxicants, and have allowed researchers to generate 'gene signatures' indicative of the changes induced by exposure (23-26). It is hoped that such signatures could be used to detect chemical exposure before clinical evidence develops, allowing both early clinical treatment if required, and immediate improvements to handling of the agents. The primary aim of the National Centre of Toxicogenomics (NCT) is to develop and populate an international reference database of gene expression data that would facilitate a knowledge base of chemical effects in biological systems (27). The NCT conducted a series of proof-of-principle experiments in which chemical-specific patterns of altered gene expression were identified across and within compound classes (23). Many studies have utilised DNA microarray technology when looking for insights into mechanisms of toxicity. An example is a study of the effects of various toxic metals on a particular epithelial cell line (28). Of the 1,200 genes examined, exposure to each different metal modified the expression of a largely unique subset of genes. In addition, different dose-dependent effects were seen on treatment with either low (noncytotoxic) or high (cytotoxic) concentrations of arsenic. Low concentrations 
modified the expression of genes for a diverse range of protein classes such as transcription factors, inflammatory cytokines, kinases and DNA repair proteins. Exposure to high concentrations did not simply amplify these changes but resulted in a very different expression profile, including induction of stress-response genes. Since the affected subsets of genes were almost completely non-overlapping, it was suggested that a threshold dose exists, above which the survival-based biological response induced by low doses shifts to the apoptotic response. These results support the development of metal-specific biomarkers of exposure and response, and demonstrate the feasibility of using expression profiling for investigation of 'safe' exposure levels.

\section{Variability in results}

It has become evident that the analysis and interpretation of the high volume of data that are produced from microarray experiments is more complicated than was originally anticipated (29). In addition, uncertainties associated with validity and reliability of results have been highlighted by comparisons between different methodologies; conclusions need to be drawn with caution (30). Studies have found inconsistencies between data from different experimental platforms based on the use of in situ synthesised short oligonucleotides, longer oligonucleotides, spotted oligonucleotides or spotted cDNAs $(31,32)$. More surprisingly, inconsistencies between results from laboratories using the same experimental platform have been found (33). Even a single dataset generated on one platform can be interpreted differently using alternative methods of data handling and analysis leading to different conclusions (34). There are many potential explanations for discrepancies, including differences in types and composition of probes, deposition techniques and labelling and hybridisation protocols (35). However, it remains uncertain which methodologies at each stage are most likely to allow detection of expression levels that are consistently representative of actual expression. Therefore, it continues to be essential to validate conclusions regarding individual genes using other technologies such as Northern blot analysis, quantitative reverse transcriptase-PCR or Western blot analysis (36).

Experimental design. The critical component of any DNA microarray experiment is the quality of the input material. Samples of cells or tissue must be treated in as reproducible a manner as possible to avoid introduction of artefactual changes in gene expression in some samples. There are a number of other important considerations concerning the input material. For example, it is often the case that samples may include heterogeneous gene expression patterns in different cell subpopulations; this is particularly true of tissue biopsies (37). The sensitivity of microarrays means that inclusion of small proportions of these subpopulations can create misleading results. This problem may be improved by advances in technology available for micro-dissection such as laser capture (38). Related to this is the use of very small samples. A technical challenge in this instance is obtaining sufficient RNA for microarray analysis. Various techniques can be used for mRNA amplification (39), although an understanding of their potential to introduce bias is important for the interpretation of results $(36,40)$.

Standardisation of data. One issue that has contributed to the apparent variability in microarray data is the difficulty in critically appraising methods and full data sets of published experiments. However, integration of microarray results into public reference databases in order to allow this appraisal presents a serious logistical challenge, particularly given that account must be taken of the differences between experimental platforms. Several organisations have engaged in standardisation to facilitate data sharing by developing compatible data formats, reporting standards and defining nomenclature (41). The Minimum Information About Microarray Experiments (MIAME) guidelines describes the reporting standards required when recording gene expression data to ensure an experiment's easy interpretation and potential to be independently verified (42). Public databases to store results from DNA microarray experiments have been developed which conform to these standards (43). It is important to recognise that while these initiatives are valuable steps forward, they only focus on reporting standards and do not address the issue of variability of the technology and how it is used.

Inferential literacy. It has been argued that to generate reproducible and valid results using microarray technology, biologists must develop improved algorithmic skills. The concept of 'inferential literacy' argues further that, in addition to algorithmic literacy, a comprehensive understanding of the nature of high throughput data, experimental design and statistical analysis is necessary for optimising progress in research using DNA microarrays (44). Realistically, it is not likely that research biologists will readily gain the required insights hence progress is likely to require improved bioinformatics and statistics support at the experimental design and interpretation stages.

\section{Additional regulatory mechanisms in gene expression}

The limitations of DNA microarray analysis discussed so far create challenges that require creative advances both in the technology and its application. However, a more fundamental criticism of the methodology concerns the relevance of mRNA expression levels in establishing the actual functional levels of proteins. The transcription of genomic DNA to mRNA is only one of many stages involved in the biosynthesis of functional proteins. Alternative splicing, translational control, post-translational modification and the sub-cellular localisation of proteins are all additional potential regulatory stages that can control the production of functional proteins (45); it is clear that mRNA levels may not necessarily correlate with protein activity. This may not be a concern for some applications; for example, whether mRNA abundances within a diagnostic mRNA signature accurately represent corresponding protein activities is not relevant as the diagnostic utility of the signature is the only important factor. However, in most research-orientated applications the aim is to identify changes in mRNA profiles that are functional in that they impact on protein expression as expected. 
Alternative splicing. Recent studies have indicated that transcripts from $40-60 \%$ of human genes are alternatively spliced (46-48), and that $70-88 \%$ of these alternative splices impact on protein function $(46,49,50)$. This suggests that splicing plays a major role in production of protein diversity and regulation of protein activity. There is increasing interest in the deregulation of splicing accuracy and the role this plays in disease. It has been estimated that $15 \%$ of all point mutations in human genetic diseases cause defects in mRNA splicing (51); an example being mutations within the tumour suppressor gene BRAC1 contributing to the development of cancer (52). Microarray technology has now evolved to allow investigation of alternative splicing and its functional importance; recent advances in array miniaturisation and in the bioinformatics of exon identification have allowed development of arrays for analysis of the expression of each individual exon (53).

Translation control. Up to $50 \%$ of human mRNAs may contain motifs capable of allowing regulation of their translation (54). It is not thought that all these genes are regulated at the level of translation at any one time, nevertheless the regulation is likely to have an important role in human physiology and pathology; for example approximately $20 \%$ of the genes regulated upon T-cell activation are affected by translation control (55). Consequently, some studies have found mRNA abundance to be a poor indicator of the levels of corresponding protein $(56,57)$. Gene-specific translational regulation involves interactions between sequences within the mRNAs, usually located in the untranslated regions, and the cellular translational machinery, specific RNA-binding proteins or specific microRNAs (54). MicroRNAs have themselves been the subject of many recent studies (58-61) since their influence on translation may be widespread, and it is interesting to note that their expression can be particularly difficult to quantify using DNA microarrays on account of their small size and complex secondary structures (62), although some microarray approaches are now available. The translational efficiency of mRNAs is likely to relate to actual protein expression more closely than the level of these mRNAs (63). A translation profiling technique has been developed in which polysome-bound mRNAs, those that are translationally active, are analysed on DNA microarrays; this may effectively integrate all levels of regulation from transcription to translation, thereby combining the potential of genomics with the physiological relevance of proteomics (64).

Post-translational mechanisms. An accurate description of the determinants of the cell phenotype would be an exhaustive quantitative and functional analysis of the proteome (65). Post-translational modifications, sub-cellular localisation, protein degradation rates and protein-protein interactions can all modify the functional levels of proteins $(66,67)$. Much of this subtlety can only be observed with proteomic analysis (1). However, proteomic techniques are generally less sensitive and are much less suitable for high throughput experiments (68), where possible mRNA and protein analyses should be seen as complementary (69).

\section{Conclusions}

The original excitement surrounding DNA microarrays has given way to a feeling in the scientific community that expectations have exceeded reality. There have been difficulties encountered in applying the technology, in achieving reproducible results and in the management of high throughput data. In addition, studies suggest that mRNA levels may reflect protein levels less frequently than had been previously recognised. However, in many cases these difficulties have been addressed. Therefore, analysis of the transcriptome using DNA microarrays may well yet fulfil its potential in a diverse range of applications.

\section{Acknowledgements}

TAH is supported by the Breast Cancer Research Action Group (UK Charity no. 1075308) and by the Breast Cancer Campaign (UK Charity no. 05074725).

\section{References}

1. Lockhart DJ and Winzeler EA: Genomics, gene expression and DNA arrays. Nature 405: 827-836, 2000.

2. Schena M, Shalon D, Davis RW and Brown PO: Quantitative monitoring of gene expression patterns with a complementary DNA microarray. Science 270: 467-470, 1995.

3. Southern EM: Detection of specific sequences among DNA fragments separated by gel electrophoresis. J Mol Biol 98: 503-517, 1975.

4. Lander ES: Array of hope. Nat Genet 21 (suppl 1): 3-4, 1999.

5. Lockhart DJ, Dong H, Byrne MC, et al: Expression monitoring by hybridization to high-density oligonucleotide arrays. Nat Biotech 14: 1675-1680, 1996.

6. Lipshutz RJ, Fodor SP, Gingeras TR and Lockhart DJ: High density synthetic oligonucleotide arrays. Nat Genet 21 (Suppl 1): 20-24, 1999

7. Fodor SP, Read JL, Pirrung MC, Stryer L, Lu AT and Salas D: Light-directed, spatially addressable parallel chemical synthesis. Science 251: 767-773, 1991 .

8. Johnson JM, Edwards S, Shoemaker D and Schadt EE: Dark matter in the genome: evidence of widespread transcription detected by microarray tiling experiments. Trends Genet 21: 93-102, 2005

9. Lobenhofer EK, Bushel PR, Afshari CA and Hamadeh HK: Progress in the application of DNA microarrays. Environ Health Perspect 109: 881-891, 2001.

10. Golub TR, Slonim DK, Tamayo P, et al: Molecular classification of cancer: class discovery and class prediction by gene expression monitoring. Science 286: 531-537, 1999.

11. Shoemaker DD and Linsley PS: Recent developments in DNA microarrays. Curr Opin Microbiol 5: 334-337, 2002.

12. Rosenwald A, Wright G, Leroy K, et al: Molecular diagnosis of primary mediastinal B cell lymphoma identifies a clinically favourable subgroup of diffuse large B cell lymphoma related to Hodgkin lymphoma. J Exp Med 198: 851-862, 2003.

13. Savage KJ, Monti S, Kutok JL, et al: The molecular signature of mediastinal large B-cell lymphoma differs from that of other diffuse large B-cell lymphomas and shares features with classical Hodgkin lymphoma. Blood 102: 3871-3879, 2003.

14. Welsh JB, Zarrinkar PP, Sapinoso LM, et al: Analysis of gene expression profiles in normal and neoplastic ovarian tissue samples identifies candidate molecular markers of epithelial ovarian cancer. Proc Natl Acad Sci USA 98: 1176-1181, 2001.

15. Dhanasekaran SM, Barrette TR, Ghosh D, et al: Delineation of prognostic biomarkers in prostate cancer. Nature 412: 822-826, 2001.

16. Martin KJ, Kritzman BM, Price LM, et al: Linking gene expression patterns to therapeutic groups in breast cancer. Cancer Res 60: 2232-2238, 2000.

17. Van't Veer LJ, Dai H, van de Vijver MJ, et al: Gene expression profiling predicts clinical outcome of breast cancer. Nature 415: 530-536, 2002. 
18. Hutchinson E: Tumour classification: recognising differences. Nat Rev Cancer 3: 716-717, 2003.

19. Feng X, Jiang Y, Meltzer P and Yen PM: Thyroid hormone regulation of hepatic genes in vivo detected by complementary DNA microarray. Mol Endocrinol 14: 947-955, 2000.

20. Yen PM: Studying hormonal regulation by microarrays: distinguishing the trees from the forest. J Clin Endocrinol Metab 90: 1241-1242, 2005

21. Kannan K, Kaminski N, Rechavi G, Jakob-Hirsch J, Amariglio N and Givol D: DNA microarray analysis of genes involved in p53 mediated apoptosis: activation of Apaf-1. Oncogene 20: 3449-3455, 2001

22. Sax JK and El-Deiry WS: p53-induced gene expression analysis. Methods Mol Biol 234: 65-71, 2003.

23. Hamadeh HK, Bushel PR, Jayadev S, et al: Gene expression analysis reveals chemical-specific profiles. Toxicol Sci 67: 219-231, 2002.

24. Burczynski ME, McMillian M, Ciervo J, et al: Toxicogenomicsbased discrimination of toxic mechanism in HepG2 human hepatoma cells. Toxicol Sci 58: 399-415, 2000.

25. Lettieri T: Recent applications of DNA microarray technology to toxicology and ecotoxicology. Environ Health Perspect 114: 4-9, 2006.

26. Waring JF, Ciurlionis R, Jolly RA, Heindel M and Ulrich RG: Microarray analysis of hepatotoxins in vitro reveals a correlation between gene expression profiles and mechanisms of toxicity. Toxicol Lett 120: 359-368, 2001.

27. Tennant RW: The National Center for Toxicogenomics: using new technologies to inform mechanistic toxicology. Environ Health Perspect 110: A8-A10, 2002.

28. Andrew AS, Warren AJ, Barchowsky A, et al: Genomic and proteomic profiling of responses to toxic metals in human lung cells. Environ Health Perspect 111: 825-835, 2003.

29. Slonim DK: From patterns to pathways: gene expression data analysis comes of age. Nat Genet 32 (Suppl): 502-508, 2002.

30. Shields R: MIAME, we have a problem. Trends Genet 22: 65-66, 2006.

31. Kuo WP, Jenssen TK, Butte AJ, Ohno-Machado L and Kohane IS: Analysis of matched mRNA measurements from two different microarray technologies. Bioinformatics 18: 405-412, 2002.

32. Grunenfelder B and Winzeler EA: Treasures and traps in genome-wide data sets: case examples from yeast. Nat Rev Genet 3: 653-661, 2002.

33. Irizarry RA, Warren D, Spencer F, et al: Multiple-laboratory comparison of microarray platforms. Nat Methods 2: 345-350, 2005.

34. Bammler T, Beyer RP, Bhattacharya S, et al: Standardizing global gene expression analysis between laboratories and across platforms. Nat Methods 2: 351-356, 2005.

35. Draghici S, Khatri P, Eklund AC and Szallasi Z: Reliability and reproducibility issues in DNA microarray measurements. Trends Genet 22: 101-109, 2006.

36. Chuaqui RF, Bonner RF, Best CJ, et al: Post-analysis follow-up and validation of microarray experiments. Nat Genet 32 (Suppl): 509-514, 2002.

37. Butte A: The use and analysis of microarray data. Nat Rev Drug Discovery 1: 951-960, 2002.

38. Simone NL, Bonner RF, Gillespie JW, Emmert-Buck MR and Liotta LA: Laser-capture microdissection: opening the microscopic frontier to molecular analysis. Trends Genet 14: 272-276, 1998

39. O'Dell DM, McIntosh TK and Eberwine JH: Single-cell molecular biology: implications for the diagnosis and treatment of neurological disease. Arch Neurol 56: 1453-1456, 1999.

40. Cole KA, Krizman DB and Emmert-Buck MR: The genetics of cancer - a 3D model. Nat Genet 21 (Suppl 1): 38-41, 1999.

41. Anonymous: Under the MIAME sun. Nat Methods 3: 415, 2006.

42. Brazma A, Hingamp P, Quackenbush J, et al: Minimum information about a microarray experiment (MIAME) - toward standards for microarray data. Nat Genet 29: 365-371, 2001.

43. Stoeckert CJ Jr, Causton HC and Ball CA: Microarray databases: standards and ontologies. Nat Genet 32 (Suppl): 469-473, 2002

44. Miron M and Nadon R: Inferential literacy for experimental high-throughput biology. Trends Genet 22: 84-89, 2006.
45. Day DA and Tuite MF: Post-transcriptional gene regulatory mechanisms in eukaryotes: an overview. J Endocrinol 157: 361-371, 1998.

46. International Human Genome Sequencing Consortium: Initial sequencing and analysis of the human genome. Nature 409: 860-921, 2001.

47. Modrek B and Lee C: A genomic view of alternative splicing. Nat Genet 30: 13-19, 2002.

48. Mironov AA, Fickett IW and Gelfand MS: Frequent alternative splicing of human genes. Genome Res 9: 1288-1293, 1999.

49. Kan Z, Rouchka EC, Gish WR and States DJ: Gene structure prediction and alternative splicing analysis using genomically aligned ESTs. Genome Res 11: 889-900, 2001.

50. Modrek B, Resch A, Grasso C and Lee C: Genome-wide analysis of alternative splicing using human expressed sequence data. Nucleic Acids Res 29: 2850-2859, 2001

51. Krawczak M, Reiss I and Cooper DN: The mutational spectrum of single base-pair substitutions in mRNA splice junctions of human genes: causes and consequences. Hum Genet 90: 41-54, 1992.

52. Liu HX, Cartegni L, Zhang MQ and Krainer AR: A mechanism for exon skipping caused by nonsense or missense mutations in BRCA1 and other genes. Nat Genet 27: 55-58, 2001.

53. Clark TA, Schweitzer AC, Chen TX, Staples MK, Lu G, Wang H, Williams A and Blume JE: Discovery of tissue-specific exons using comprehensive human exon microarrays. Genome Biol 8: R64, 2007.

54. Hughes TA: 5' untranslated regions: critical regulators of capdependent translation. In: Leading-Edge Messenger RNA Research Communications. Ostrovskiy MH (ed). Nova Science Publishers, New York, pp16-29, 2007.

55. Garcia-Sanz LA, Mikulits W, Livingstone A, Lefkovits I and Mullner EW: Translational control: a general mechanism for gene regulation during T cell activation. FASEB J 12: 299-306, 1998.

56. Anderson L and Seilhamer J: A comparison of selected mRNA and protein abundances in human liver. Electrophoresis 18: 533-537, 1997.

57. Gygi SP, Rochon Y, Franza BR and Aebersold R: Correlation between protein and mRNA abundance in yeast. Mol Cell Biol 19: 1720-1730, 1999.

58. Valencia-Sanchez MA, Liu J, Hannon GJ and Parker R: Control of translation and mRNA degradation by miRNAs and siRNAs. Genes Devel 20: 515-524, 2006

59. Storz G, Altuvia S and Wassarman KM: An abundance of RNA regulators. Ann Rev Biochem 74: 199-217, 2005.

60. Gottesman S: The small RNA regulators of Escherichia coli: roles and mechanisms. Ann Rev Microbiol 58: 303-328, 2004.

61. Nakamoto M, Jin P, O'Donnell WT and Warren ST: Physiological identification of human transcripts translationally regulated by a specific microRNA. Hum Mol Genet 14: 3813-3821, 2005.

62. Hu Z, Zhang A, Storz G, Gottesman S and Leppla SH: An antibody-based microarray assay for small RNA detection. Nucleic Acid Res 34: E52, 2006.

63. Zong Q, Schummer M, Hood L and Morris DR: Messenger RNA translation state: the second dimension of high-throughput expression screening. Proc Natl Acad Sci USA 96: 10632-10636, 1999.

64. Pradet-Balade B, Boulme F, Beug H, Mullner EW and Garcia-Sanz JA: Translation control: bridging the gap between genomics and proteomics? Trends Biochem Sci 26: 225-229, 2001.

65. Anderson NL and Anderson NG: Proteome and proteomics: new technologies, new concepts, and new words. Electrophoresis 19: 1853-1861, 1998.

66. Winssinger N, Ficarro S, Schultz PG and Harris JL: Profiling protein function with small molecule microarrays. Proc Natl Acad Sci USA 99: 11139-11144, 2002.

67. Kirschner M: Intracellular proteolysis. Trends Cell Biol 9: M42-M45, 1999.

68. Dove A: Proteomics: translating genomics into products? Nat Biotech 17: 233-236, 1999.

69. Celis JE, Kruhoffer M, Gromova I, et al: Gene expression profiling: monitoring transcription and translation products using DNA microarrays and proteomics. FEBS Lett 480: 2-16, 2000 . 